\title{
ESTUDO CINÉTICO E TERMODINÂMICO DA PURIFICAÇÃO DO GLICEROL POR ADSORÇÃO
}

\author{
I. B. JULIÃO ${ }^{1}$, G. N. SCHUABB ${ }^{1}$, A. P. $\operatorname{ALVES}^{1}$ e M. F. MENDES ${ }^{1}$ \\ ${ }^{1}$ Universidade Federal Rural do Rio de Janeiro, Departamento de Engenharia Química, \\ Laboratório de Termodinâmica Aplicada e Biocombustíveis \\ E-mail para contato: marisamf@ufrrj.br
}

\begin{abstract}
RESUMO - O aumento na produção de glicerol, nos últimos anos, se deu pelas constantes pesquisas por fontes alternativas de energia, gerando uma demanda na busca por novas tecnologias para sua purificação. Entre os vários processos de separação, a adsorção se apresenta como uma opção favorável para a remoção dos contaminantes do glicerol, devido ao seu baixo custo e simplicidade de operação. Diante disso, esse trabalho propõe o estudo cinético e termodinâmico do processo de adsorção para purificação do glicerol, utilizando diferentes adsorventes, sendo eles: Argila clarificante Pure-Flo Supreme B81, carvão ativado e terra diatomácea. Para isso, foi utilizada uma solução sintética de glicerol, constituída de glicerina PA e óleo de soja. Os resultados obtidos em diferentes temperaturas mostram que os dados experimentais foram bem relacionados com as isotermas de Langmuir, Freundlich e Redlich-Peterson. Os valores negativos da variação da energia livre de Gibbs indica que a adsorção do óleo de soja é um processo espontâneo, enquanto que a entalpia, negativa para a argila e o carvão, e positivo para a terra, indicam que o processo de adsorção é endotérmico e exotérmico, respectivamente.
\end{abstract}

\section{INTRODUÇÃO}

Nos últimos anos tem-se observado o crescente interesse em estudos aplicados às energias alternativas em detrimento das provenientes do petróleo, devido às mudanças climáticas e aos impactos ambientais. Combustíveis como o biodiesel tem se mostrado atraentes, devido ao seu apelo ambiental, podendo ser produzidos utilizando óleos vegetais, de fritura ou gordura animal. Diante disso, o biodiesel é produzido pela reação de transesterificação de óleos vegetais ou gordura animal (SILVA et al., 2009). O aumento na produção de biodiesel aumenta a produção de glicerol como subproduto, ocasionando na desvalorização do mercado (DASARI et al., 2005).

O glicerol pode apresentar impurezas diversas, como: álcool não reagido, água, sais inorgânicos (provenientes do catalisador não reagido), óleo de soja não hidrolisado, ácidos graxos livres, entre outras impurezas oriundas da matéria prima, as quais diminuem o preço do produto já que inviabilizam sua utilização em diversas indústrias. Para tal, seria necessário um processo de purificação; todavia o mesmo é geralmente caro, inviabilizando certas produções (CHUN-HUI et al., 2008). 
Para purificar o glicerol, a adsorção é uma escolha viável ao ser comparada com outros métodos de refinamento, como a destilação simples, extração com solventes polares, troca iônica, entre outros, por apresentar baixo custo, ser de fácil execução e utilizar equipamentos simples, além de possuir a possibilidade da recuperação do adsorvente gasto, alta eficiência e seletividade (KUMAR et al., 2010).

Portanto, o objetivo desse trabalho é avaliar o processo de purificação do glicerol proveniente da produção de biodiesel utilizando a técnica de adsorção através de uma solução sintética de glicerina P.A. contaminada com óleo de soja, comparar a eficiência de diferentes adsorventes e a influência das impurezas oriundas da produção.

\section{MATERIAIS E MÉTODOS}

\subsection{Materiais}

A solução de glicerina sintética foi preparada em laboratório utilizando glicerina P.A. (VETEC QUÍMICA FINA Ltda, RJ), e óleo de soja comercial. Os três adsorventes utilizados foram: carvão ativado (VETEC QUÍMICA FINA Ltda., RJ), argila clarificante Pure-Flo Supreme B81, cuja matéria prima consiste em hormita e esmectita (ABOISSA ÓLEOS VEGETAIS Ltda.) e terra diatomácea, material residual do processo filtração de cerveja, que consiste basicamente de sílica hidratada, adquirido de uma indústria cervejeira.

\subsection{Metodologia Experimental}

Tratamento do material adsorvente: Inicialmente, os adsorventes receberam um tratamento térmico, a fim de melhorar a capacidade de adsorção. A argila e o carvão foram levados à estufa (DeLeo, tipo A35E) por 24 horas a $105^{\circ} \mathrm{C}$, para a eliminação de umidade. A terra diatomácea foi levada à mufla (Quimis, modelo Q318S25T) a $800{ }^{\circ} \mathrm{C}$ para ser calcinada, a fim de eliminar quaisquer resíduos orgânicos.

Experimentos para análise cinética e termodinâmica: Os experimentos de adsorção foram realizados utilizando a solução sintética para o estudo da cinética e da termodinâmica do processo de adsorção com os três adsorventes, variando-se o tempo de contato e a temperatura dos ensaios, sendo os mesmos realizados em triplicata.

Inicialmente, foram realizados ensaios de cinética para a determinação do tempo de equilíbrio. Erlenmeyers de $125 \mathrm{~mL}$ com, aproximadamente, $2,5 \mathrm{~g}$ do adsorvente e $30 \mathrm{~g}$ de solução sintética, foram colocados na mesa agitadora (Orbital Digital Shaker-Daiki-80-283) à temperatura de $40{ }^{\circ} \mathrm{C}$ (banho ultra termostático, LAB 100-SKO180-Pro4) e agitação de 250 rpm. Em seguida, o conteúdo de cada frasco foi filtrado utilizando-se uma bomba de vácuo. Alíquotas foram retiradas de $10 \mathrm{em} 10 \mathrm{~min}$ para serem analisadas a fim de se obter a concentração de glicerol no equilíbrio.

As análises das alíquotas foram realizadas no Laboratório de Síntese Orgânica do Departamento de Química da UFRRJ, utilizando um espectrofotômetro (SHMADZU, modelo UV mini 1240 UV-Vis). 
Com o tempo de equilíbrio determinado, com base em experimentos prévios utilizando um planejamento experimental (ALVES, 2017), os valores de concentração inicial de glicerol (70\%) e quantidade de adsorvente $(1,2 \mathrm{~g})$ foram fixados em seu ponto ótimo para melhor purificação do glicerol (acima de 90\%). Então, seguiu-se com os experimentos variando a temperatura $\left(25,32,5\right.$ e $\left.40{ }^{\circ} \mathrm{C}\right)$ e o tempo de residência ( $\left.15 \mathrm{a} 60 \mathrm{~min}\right)$, retirando-se alíquotas de $15 \mathrm{em} 15 \mathrm{~min}$. Esses experimentos ocorreram da mesma forma dos anteriores. Em todos os experimentos, a porcentagem de remoção das impurezas da glicerina foi realizada por espectrofotometria na região do UV/visível no comprimento de $279 \mathrm{~nm}$.

\section{RESULTADOS E DISCUSSÃO}

\subsection{Cinética de Adsorção}

A Figura 1 apresenta os dados experimentais dos ensaios de cinética, os quais foram realizados com a solução sintética de glicerina P.A. e óleo de soja, com os três adsorventes diferentes, em diferentes temperaturas. Esse estudo permitiu obter o tempo de equilíbrio para se atingir a capacidade máxima de adsorção dos adsorventes, assim como a cinética do processo.

A partir dos gráficos é possível visualizar que o tempo necessário para os sistemas entrarem em equilíbrio são inferiores à 60 min para todos os adsorventes, e que a remoção de óleo de soja diminuiu com o tempo de contato até o equilíbrio. Para a argila, o tempo de equilíbrio foi atingido em 30 min à $25^{\circ} \mathrm{C}$ e em 60 min para 32,4 e $40{ }^{\circ} \mathrm{C}$, apresentando uma remoção de $73,08 \%$. Para a terra de diatomácea, em todas as temperaturas, o tempo de equilíbrio foi de 30 min, com uma remoção máxima de 70,33\%. Para o carvão ativado, tem-se uma remoção máxima de $88,29 \%$ no tempo de $60 \min$ à $25^{\circ} \mathrm{C}$.

É interessante apontar que na terra diatomácea, passados 30 min de adsorção, em todas as temperaturas, ocorreu a diminuição do teor de glicerol, indicando a adsorção do glicerol e não mais do contaminante. $\mathrm{O}$ mesmo pode ser observado com a argila à $25^{\circ} \mathrm{C}$ no tempo de 30 min, e com o carvão à 32,4 e $40^{\circ} \mathrm{C}$ após 45 min.

Figura 1 - Cinética da adsorção da solução sintética em diferentes temperaturas da: (a) argila clarificante, (b) terra diatomácea e (c) carvão ativado
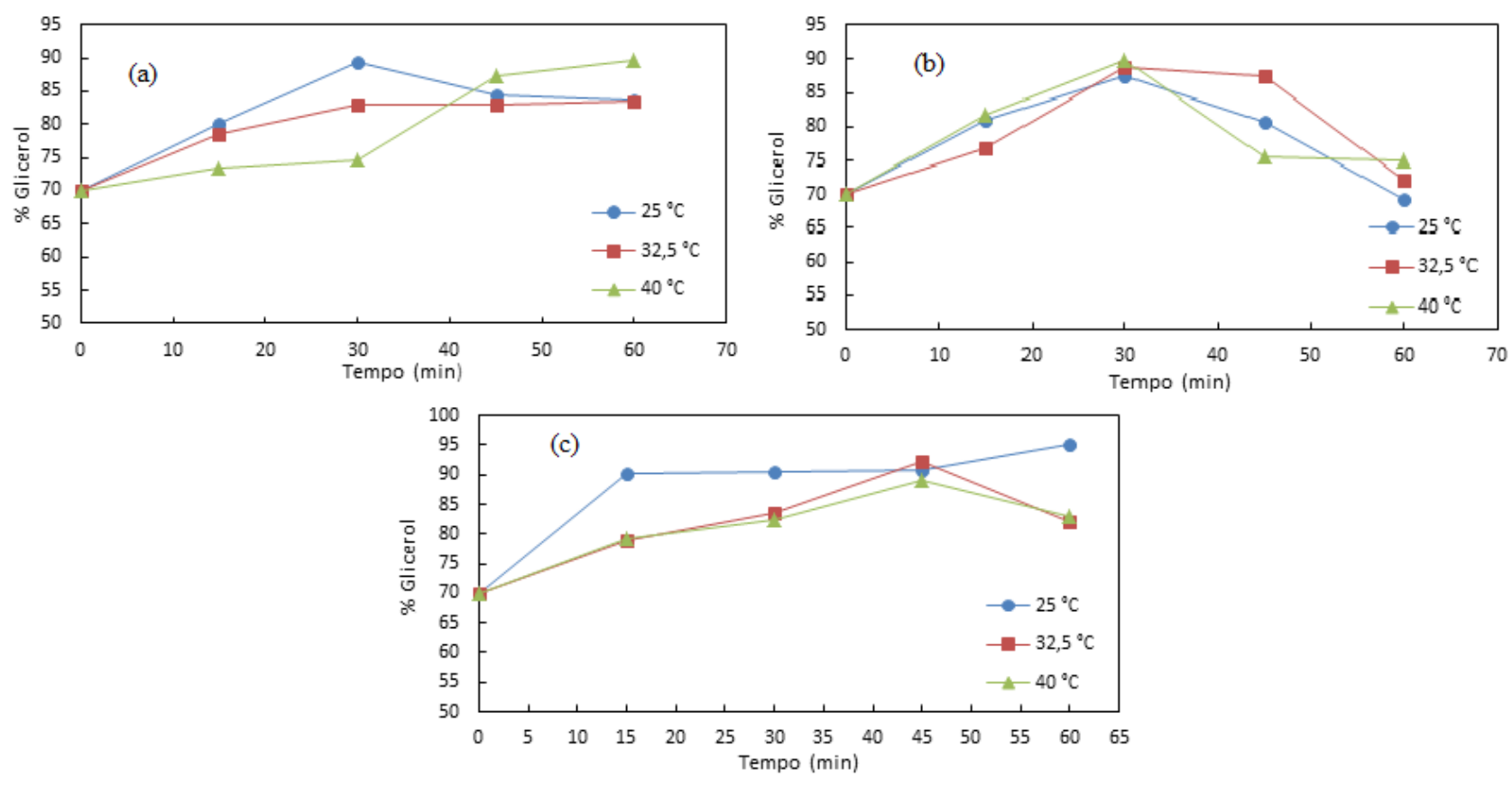


\subsection{Avaliação dos parâmetros Termodinâmicos da adsorção}

Os valores dos parâmetros termodinâmicos da adsorção do óleo de soja estão, usando os três adsorventes, estão apresentandos na Tabela 1.

Tabela 1 - Parâmetros termodinâmicos da adsorção

\begin{tabular}{|c|c|c|c|c|c|}
\hline \multirow[t]{2}{*}{ Adsorvente } & \multirow{2}{*}{$\begin{array}{c}\Delta \mathbf{H} \\
\left(\mathrm{J}^{\prime} \cdot \mathrm{mol}^{-1}\right)\end{array}$} & \multirow{2}{*}{$\begin{array}{c}\Delta S \\
\left(\mathbf{J}^{\prime} \mathbf{m o l}^{-1} \cdot \mathbf{K}^{-1}\right)\end{array}$} & \multicolumn{3}{|c|}{$\Delta G\left(J^{\prime} \cdot m^{-1}\right)$} \\
\hline & & & $298,15 \mathrm{~K}$ & $305,65 \mathrm{~K}$ & $313,15 \mathrm{~K}$ \\
\hline Argila & $-73306,7$ & $-114,1$ & $-39274,0$ & $-38417,9$ & $-37561,8$ \\
\hline Terra & 6874,6 & 153,1 & $-38760,8$ & $-39908,8$ & $-41056,7$ \\
\hline Carvão & $-14316,5$ & 85,1 & $-39684,9$ & $-40323,0$ & $-40961,2$ \\
\hline
\end{tabular}

Em todos os processos de adsorção estudados, os valores negativos de $\Delta \mathrm{G}$ indicam uma redução na energia livre de Gibbs $(\Delta \mathrm{G})$, como esperado para um processo espontâneo e favorável. Com o carvão e a terra houve uma diminuição nos valores de $\Delta \mathrm{G}$ com o aumento da temperatura, indicando uma maior força motriz, ocasionando em uma maior afinidade da adsorção em temperaturas mais elevadas. Para a argila, o processo é mais favorecido à temperatura ambiente, já que se tem uma diminuição dos valores de $\Delta \mathrm{G}$.

Os valores negativos de entalpia para a argila e carvão indicam que o processo de adsorção é exotérmico, enquanto o valor positivo para a terra indica que o processo foi endotérmico. A variação de entropia $\Delta \mathrm{S}$ indica o nível de desordem do sistema. Quanto mais randômico o sistema, maior sua entropia e maior a afinidade entre o adsorvente e a impureza. Portanto, os valores positivos de $\Delta \mathrm{S}$ indicam que as moléculas do óleo de soja se encontram mais desordenadas adsorvidas do que livres em solução (BEREKET et al., 1997; AKSU \& TEZER, 2004).

\subsection{Isotermas de Adsorção}

A adsorção pode ser avaliada através de suas isotermas, geralmente, utilizadas para descrever a relação entre a concentração de adsorvato em solução e adsorvente, a temperatura constante, assim como uma análise do processo de adsorção, indicando a eficiência do mesmo. Para melhor entendimento do processo, foi realizada uma comparação quantititativa das isotermas correlacionando os dados experimentais às equações de Freundlich, Langmuir e Redlich-Peterson.

Para os três modelos, Freundlich, Langmuir e Redlich-Peterson, os dados experimentais foram bem representados e indicaram uma grande afinidade entre os adsorventes e os contamiantes (AKSU e TEZER, 2004). A isoterma de Langmuir foi a que teve o melhor ajuste entre os três modelos, indicando que a adsorção ocorre em monocamada. A Figura 2 apresenta o comportamento das isotermas para os experimentos realizados com a argila, e as Tabelas 2, 3 e 4 apresentam as constantes e os coeficientes de correlação dos modelos. 
Figura 2 - Isotermas de (a) Langmuir, (b) Freundlich e (c) Redlich-Peterson relativas ao processo de adsorção de óleo de soja usando a argila clarificante
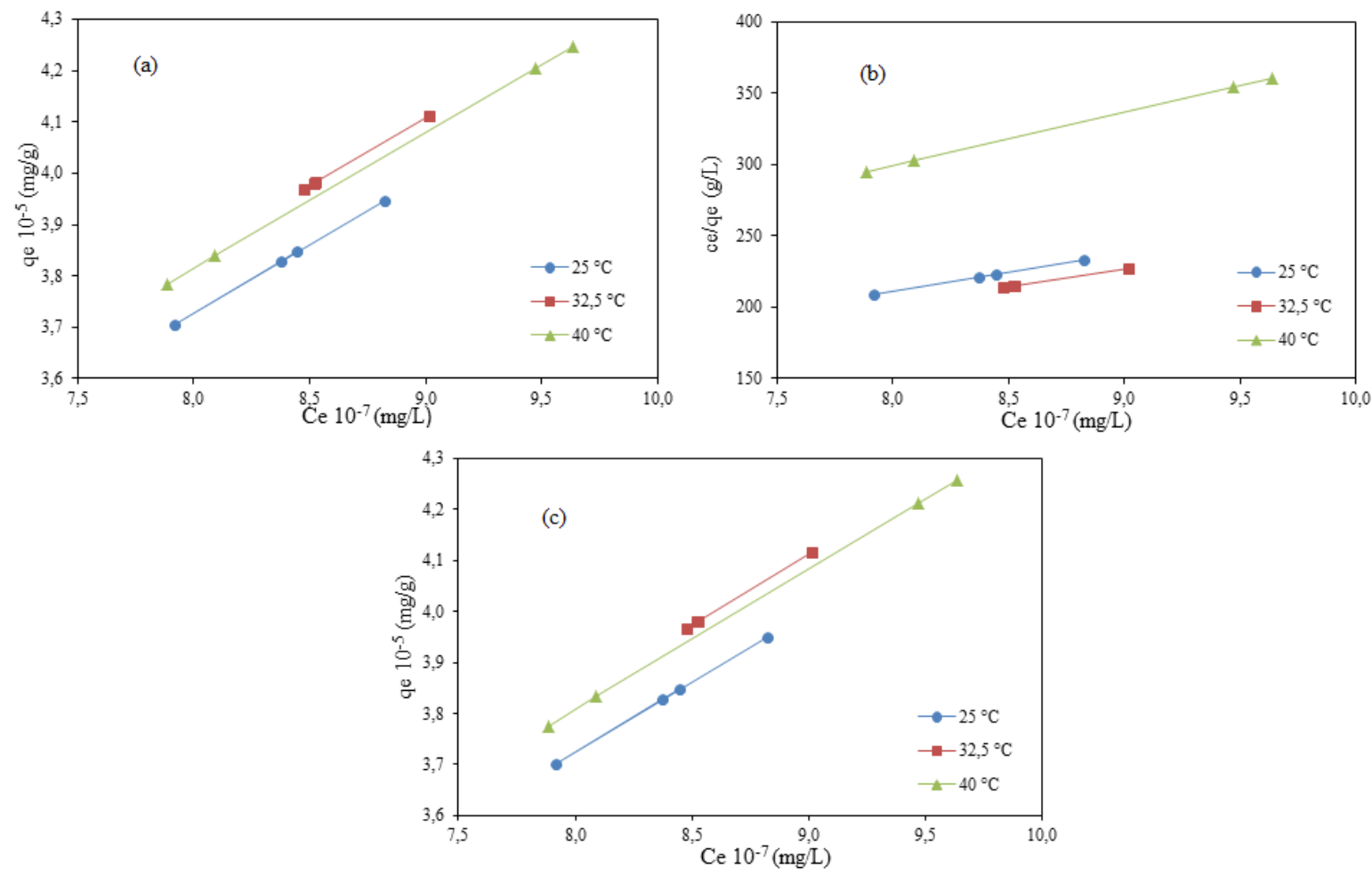

Tabela 2 - Constantes das isotermas de Langmuir, Freundlich e Redlich-Peterson para a argila

\begin{tabular}{ccccccccccc}
\hline $\begin{array}{c}\text { Temperatura } \\
\left({ }^{\circ} \mathbf{C}\right)\end{array}$ & \multicolumn{3}{c}{ Langmuir } & \multicolumn{3}{c}{ Freundlich } & \multicolumn{3}{c}{ Redlich-Peterson } \\
\hline & $\mathbf{q}$ & $\mathbf{K}_{\mathbf{L}}$ & $\mathbf{R}^{\mathbf{2}}$ & $\mathbf{\mathbf { K } _ { \mathbf { F } }}$ & $\boldsymbol{n}$ & $\mathbf{R}^{\mathbf{2}}$ & $\mathbf{K}_{\mathbf{R}}$ & $\boldsymbol{\alpha}$ & $\boldsymbol{\beta}$ & $\mathbf{R}^{\mathbf{2}}$ \\
25 & 379014,8 & 398024,5 & 0,96418 & 9,759516 & 1,724782 & 0,96256 & 0,719459 & 0,100000 & 0,403173 & 0,96231 \\
32,5 & 397556,0 & 369729,9 & 0,99963 & 10,28010 & 1,728573 & 0,99954 & 0,706252 & 0,100000 & 0,400571 & 0,99954 \\
40 & 267300,2 & 33558525 & 0,69403 & 10,78451 & 1,737397 & 0,68653 & 0,706600 & 0,100000 & 0,400960 & 0,68313 \\
\hline
\end{tabular}

Tabela 3 - Constantes das isotermas de Langmuir, Freundlich e Redlich-Peterson para a terra diatomácea

\begin{tabular}{ccccccccccc}
\hline $\begin{array}{c}\text { Temperatura } \\
\left({ }^{\circ} \mathbf{C}\right)\end{array}$ & \multicolumn{3}{c}{ Langmuir } & \multicolumn{3}{c}{ Freundlich } & \multicolumn{4}{c}{ Redlich-Peterson } \\
\hline & $\mathbf{q}_{\mathbf{m}}$ & $\mathbf{K}_{\mathbf{L}}$ & $\mathbf{R}^{\mathbf{2}}$ & $\mathbf{\mathbf { K } _ { \mathbf { F } }}$ & $\boldsymbol{n}$ & $\mathbf{R}^{\mathbf{2}}$ & $\mathbf{K}_{\mathbf{R}}$ & $\boldsymbol{\alpha}$ & $\boldsymbol{\beta}$ & $\mathbf{R}^{\mathbf{2}}$ \\
25 & 373661,2 & 394499,5 & 0,94970 & 9,814536 & 1,724323 & 0,91164 & 0,710982 & 0,100000 & 0,402024 & 0,91101 \\
32,5 & 340417,0 & 314887,2 & 0,78829 & 10,70532 & 1,756674 & 0,95012 & 0,708025 & 0,100000 & 0,398124 & 0,78521 \\
40 & 209327,5 & 131201,7 & 0,88151 & 10,97465 & 1,836677 & 0,78279 & 0,813111 & 0,100000 & 0,439020 & 0,78192 \\
\hline
\end{tabular}

Tabela 4 - Constantes das isotermas de Langmuir, Freundlich e Redlich-Peterson para o carvão ativado

\begin{tabular}{ccccccccccc}
\hline $\begin{array}{c}\text { Temperatura } \\
\left({ }^{\circ} \mathbf{C}\right)\end{array}$ & \multicolumn{3}{c}{ Langmuir } & \multicolumn{3}{c}{ Freundlich } & \multicolumn{3}{c}{ Redlich-Peterson } \\
\hline & $\mathbf{q}$ & $\mathbf{K}_{\mathbf{L}}$ & $\mathbf{R}^{\mathbf{2}}$ & $\mathbf{K}_{\mathbf{F}}$ & $\boldsymbol{n}$ & $\mathbf{R}^{\mathbf{2}}$ & $\mathbf{\mathbf { K } _ { \mathbf { R } }}$ & $\boldsymbol{\alpha}$ & $\boldsymbol{\beta}$ & $\mathbf{R}^{\mathbf{2}}$ \\
25 & 590776,2 & 2117260 & 0,98989 & 11,81410 & 1,67500 & 0,98227 & 0,163852 & 3,420299 & 0,098005 & 0,97928 \\
32,5 & 343430,7 & 8381141 & 0,95192 & 10,76036 & 1,728914 & 0,80249 & 0,619250 & 0,100000 & 0,400384 & 0,94962 \\
40 & 350936,6 & 358484,6 & 0,96705 & 10,71234 & 1,754685 & 0,96623 & 0,615487 & 0,100000 & 0,399337 & 0,96588 \\
\hline
\end{tabular}




\section{CONCLUSÃO}

Os dados experimentais, obtidos com a ajuda de um planejamento prévio, indicaram uma ótima remoção do óleo de soja, atingindo um teor máximo de glicerol de 95,59\%, $97,25 \%$ e $90,26 \%$ com o carvão, terra diatomácea e argila, respectivamente. Todas as isotermas apresentaram um bom comportamento; o tempo de contato para atingir o equilíbrio foi inferior a 60 min para todos os adsorventes. Todos os processos de adsorção se mostraram espontâneos. Além disso, observou-se que para a argila e o carvão, a capacidade adsortiva diminui com o tempo, uma vez que se trata de um processo exotérmico, enquanto para a terra diatomácea tem-se um processo endotérmico.

\section{REFERÊNCIAS BIBLIOGRÁFICAS}

AKSU, Z., TEZER, S. Equilibrium and Kinetic Modelling of Biosorption of Remazol Black B by Rhizopus arrhizus in a batch System: Effect of Temperature. Proc. Biochemistry, v. 36, p. 431-439. 2004.

ALVES, A. P. Purificação de glicerol utilizando diferentes adsorventes: Análise técnica e termodinâmica. 2017. 120p Dissertação (Mestrado em Engenharia Química, Tecnologia Química). Instituto de Tecnologia, Departamento de Engenharia Química, Universidade Federal Rural do Rio de Janeiro, Seropédica, RJ, 2017.

BEREKET, G.; AROGUZ, A. Z.; ÖZEL, M. Z. Removal of Pb (II), Cd (II), and Zn (II) from aqueous solutions by adsorption on bentonite. Journal of Colloid and Interface Science, v. 187, p. 338-343. 1997.

CHUN-HUI, Clayton Z.; BELTRAMINI, Jorge N.; FAN, Yong-Xian; LU, Max G. Q. Chemoselective Catalytic Conversion of Glycerol as a Biorenewable Source to Valuable Commodity Chemicals. Chemical Society Reviews. v. 37, p. 527-549. 2008.

DASARI, M. A.; KIATSIMKUL, P. P.; SUTTERLIN, W. R.; SUPPES, G. J.; Low-pressure hydrogenolysis of glycerol to propylene glycol. Applied Catalysis A: General. p.281, 225. 2005.

KUMAR, P.S., RAMALINGAM, S., SENTHAMARAI, C., NIRANJANAA, M., VIJAYALAKSHMI, P., SIVANESAN, S. Adsorption of dye from aqueous solution by cashew nut shell: Studies on equilibrium isotherm, kinetics and thermodynamics of interactions. Desalination. v. 261, p. 52-60. 2010.

SILVA, G. P.; MACK, M.; CONTIERO, J., Glycerol: A promising and abundant carbon source for industrial microbiology. Biotechnology advances. v. 27, p.3 0-39. 2009. 